\title{
Idea of Knowledge-Based Engineering using CAD Model
}

\author{
Bian Xiuwu Maochun \\ School of Environmental Science, University of Science and Technology of China, P.R.China. \\ maochun3442@128mail.com
}

\author{
ArticleInfo \\ Journal of Machine and Computing (http://anapub.co.ke/journals/jmc/jmc.html) \\ Doi : https://doi.org/10.53759/7669/jmc202101024
}

Received 30 March 2021; Revised form 30 June 2021; Accepted 18 August 2021; Available online 05 October 2021. (O2021 The Authors. Published by AnaPub Publications.

This is an open access article under the CC BY-NC-ND license. (http://creativecommons.org/licenses/by-nc-nd/4.0/)

\begin{abstract}
Manufacturing firms have been compelled to invest heavily in digitizing and optimizing their technical and manufacturing operations as a result of mass customization. When developing and introducing new goods, not only must manufacturing procedures be computerized, but also information of how the products must be developed and manufactured based on client needs must be applied. One major academic issue is to assist the industry in ensuring that stakeholders understand the background information of automated engineering all through the production process. The goal of the study described in this article is to provide a foundation for a connectivity perspective of Knowledge-Based Engineering (KBE). The use of graph theory in conjunction with content-based filtering methods is used to handle network creation and contextualization, which are fundamental ideas in connectivism. To enable a connectivity management culture, the article demonstrates how engineering information in spreadsheet, knowledge representation, and Computer Aided Design (CAD) models may be infiltrated and displayed as filtering graphs.
\end{abstract}

Keywords - Computer Aided Design (CAD), Knowledge-Based Engineering (KBE), CAD, Data Management.

\section{INTRODUCTION}

Engineering education focuses on skills and information that engineers use while designing goods and manufacturing processes. This is a wide definition with a focus on application, implying that information is used in decision-making and therefore eliminates curiosity. The rationale for "whom, what, where, when, how and why" anything is to be done or formed is referred to as engineering knowledge. Product knowledge is the total of engineering knowledge officially expressed for one product, and it can be found in all accessible depictions of the good or service. The constant pushing factor for capturing and automatically using such technical knowledge has been mass customisation. Many businesses, for example, have invested considerable resources in parametrizing (almost "automating") CAD models in order to react rapidly and correctly to changes in the production needs and requirement. As a consequence, rather of creating single items, engineers are increasingly concentrating on building product's groupings with more flexible and wider designing space. Organizational learning and knowledge engineering (KBE) have been attempting to collect, synthesize, and streamline the use of this type of expertise within manufacturing and production design for generations, in tandem to discretized CAD models [1].

Much though KBE systems have received a lot of attention in the past several decades, businesses still find them difficult to create and even more difficult to sustain over time. It is recommended that a connectivistic perspective of knowledge be taken in order to participate in how it may assist industries in maintaining their product knowledge throughout time. The following is how the paper is structured: Section II focuses on the reference model where aspects of product knowledge and codification; and Interconnectionism, and filtering and graphs have been evaluated. Section III focuses on dissecting the components of the technical knowledge in parametric design. Lastly, Section IV concludes the paper.

\section{REFERENCE MODEL}

We will examine how corporate understanding is typically represented in this part, followed by a research on connectivism, a viewpoint on expertise that has guided the creation of the suggested working methodology, which is backed up by tools and techniques that are also given in this article. The connectivistic viewpoint is discussed in terms of universal learning and knowledge. Finally, brief explanations to computational geometry and filtering are provided as background material for the next parts.

\section{Product Knowledge and Codification}

Variants between the synthesizing and evaluation stages are used to address architectural design issues. The criteria are compared to design solutions generated via creative methods. Most of the trial - and - error cycles that occur throughout new product development procedures are part of the learning process of how to address the product's issues. Product knowledge refers to the information gained and codified throughout these trials. As the product develops, a collection of proven solutions will emerge, which will be evaluated when a client requests the products for novel criterion set [2]. 
Whenever remedies have been analyzed, the synthesis stage changes to start searching the present frameworks, which can be integrated to mitigate novel challenges. Moreover, following the development of products, the testing process of it against criteria becomes more codified, and it may sometimes be avoided due to inductive reasoning. In other words, based on previous experience, it is possible to infer that the new approach will work. Knowledge that has been proven and codified may be used as a design basis. The procedures for creating a variation are clearly defined and based on user needs within the mature phase of the widely-configured products. In this stage, computer assistance may be used extensively in the synthesizing and general framework, which is known as knowledge-based engineering (KBE).

KBE represents a technique that synthesizes the proposals of designs, which have been identified as a science oriented onto using software tool capable of capturing and systematically reusing commodity and procedure engineering expertise, with an ultimate objective of minimizing the development timeframe and costs of products by automating manual as well as non-creative planning phase, as well as assistance for interdisciplinary optimal design in all the stages of the product design process. Because there are no obvious methods to test any potential approach, the states of the issue are difficult to describe, and the understanding of the difficulties is difficult to capture and communicate, engineering challenges are usually ill-structured.

Because of this basic characteristic of engineering issues, artificial intelligence has been used to streamline design engineering procedures. KBE is traditionally built on a knowledge-based intelligent system, with production rules serving as the main carriers of collected information. As long as it can be stated to be the integration of machine learning and computer-aided design, KBE may be considered an interdisciplinary joint mixing of fields, as shown in Fig 1 . Computeraided design comprises methodologies to design geometries and products structures, whereas machine learning is the gathering of processes and modeling using the concept of computer engineering research segment, which allows for more flexible form of modeling of inclusive processes and ideas for logic.

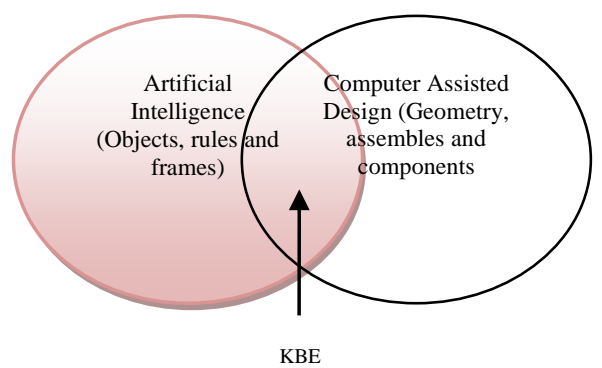

Fig 1. KBE interdisciplinary joint

From a technological standpoint, KBE systems are computer programs that include expertise and logic processes, as well as geometric management abilities, to offer advanced engineering alternatives. Furthermore, KBE can be visualized in the scope of knowledge formulation, and $\mathrm{KBE}$ represents the subset of more predictive modeling that represents a subdivision of data management. CAD models constitute component of the documented technical knowledge, as may be seen from such definitions. They emerge from the application of technical skillset and identify the manner in which products will be embodied and developed [3]. The regulations and logic can be integrated within the Design model, but they can also be kept in workbooks or reference stores that contain facts and rules.

\section{Interconnectionism}

Experts have conceptualized constructivist approach as a theory of knowledge. It is described as "the concept that knowledge is dispersed over a web of contacts," and it addresses learning that takes place inside technology and organizations, which KBE is ultimately designed to assist. Constructivist approach is founded on nine concepts (which academics have articulated but not numbered):

1. To show the whole and to allow selection of the optimal method, methods of learning necessitate a variety of viewpoints

2. Learning is the process of linking distinct nodes or knowledge sources to create a network.

3. Knowledge is distributed via networks.

4. Technology enables and facilitates learning by storing knowledge in non-human equipment.

5. The capacity to learn more that the understood elements.

6. Learning and comprehension are processes that are progressive (not products or endstates)

7. Individuals now need to be able to identify connections, detect the relevant pattern, and evaluate the concepts, ideas and fields.

8. All connectivistic training actions are designed towards currency (correct, novel dataset)

9. The protocol of making judgments in a form of learning.

The importance of data received and the choice about what to understand are visualized via lenses of constantly changing reality. Whereas a more applicable response can be obtained in the present age, it might not be applicable in the future generation due to the available iterations within the metadata environment influencing the aspects of decisionmaking. Within constructivist theory, five components have been found [4]. Knowledge is stored in networks. Individualized filters and the meaning of knowledge are shaped by the interaction of conduit, context, and content. The subsections that follow provide an overview of these elements. 


\section{Networks}

Knowledge is a connectivity creation process, according to the connectivistic view of learning. This is achieved in the cognitive technology, via communities of practices, computer-assisted collaborative work platforms, taxonomies, and information webs. These technologies, however, are not regarded to be core of a knowledge-based engineering project.

\section{The Contexts}

Emotional responses, recent events, attitudes, and the environmental elements are all included in the connectivistic perspective of contexts. Each piece has properties that, when seen in a particular way, reveal what is conceivable in the debate [5]. The structure of the discussion, paradigm, or pattern of thinking is linked to the item. The context-game involves the creation and articulation of what is acceptable and valued, as well as the norms to that we will resort in conflict situations. Persons, politics, acceptable ways seeing and experiencing, current events, company history, and a variety of other variables all play a role in the frame of reference of adopting a new business strategy, KBE, Knowledge Engineering (KE) and Knowledge Management (KM) theories do not usually include context in this wide sense. However, context has an impact on how knowledge is applied in Knowledge management and how stakeholders understand it.

\section{Conduit}

Intellect (i.e., professionals) and seekers (i.e., data users) interact via conduits, and the known thing participate more actively through them. The facilities that make information relevant, current, and accessible are known as conduits. PLMsystems, intranets, blogging, and shared data servers are examples of today's conduits in industrial firms.

\section{Filters}

In [6], authors gives a short overview of how knowledge has been used in the past, concluding that we sometimes go to one resource for a few items of data (for instance newspapers). We now consult a thousand multiple sources of data to develop our own opinions. He goes on to argue that humans have evolved into the network's filter, mediator, and weaver. Because human beings are constrained when it comes to their ability to focus (we might just consider completing a single assignment within a specific timeframe; moreover, we have a limited timeframe in a single day). In addition, the massive set of data and logic is constantly developing on a daily basis, which makes it essential to filter text categorization on individualized filtration and also governance mechanism.

\section{Provide Information}

Of course, the most important element is the substance, even though it may be argued that training ability is fundamental compared to what we already know. Application, on the contrary, is decided by more than just the content's personality. Maintaining data currency is critical for effective knowledge growth and function management. Material must blend in with the environment and conduit suggesting that the content must be nearer. Knowledge engineers presently spend a lot of time searching for content, but the content should be searching for them.

\section{Filtering and Graphs}

Edges (E) and Nodes (N) make up the grid Structure (N, E) (E). The connections show how the node is linked as itemsets of pair of nodes, with parent unit at the top and the destination address at the bottom. Neighbours refer to two vertices that are linked with edges. The amount of neighbours a cluster possesses, or even how many connections go into and out of it, determine its degree. The number of nodes relies on (parents) is the in-degree, whereas the number of nodes it leans on is out-degree (i.e. the child). Access points \& connections may be labelled to indicate what sort of entity they reflect and what kind of connection they constitute. Nodes and edges may also have attributes added to them.

The state of the displayed item is reflected in node characteristics, while the state of the connection between two nodes is reflected in edge attributes. Filtration is the technique of creating diagrams by minimizing or combining collections employing set theories in this article. Use the basic set of Logical functions (union, distinction, and segment) on vertices and edges to build searches. SQL can be used to filter underlying data that is stored in a relational database. However, an increasing number of database systems and graphs querying languages are being developed, each with a different set of graph theory-based methods for maintaining and filtering huge networks. The latter technique of data storing outperforms database systems when the data is closely linked. When the relationships between both the documented items are as significant as or more significant than the units themselves, database systems are appropriate. In the examples shown in this paper, Neo4j was used to store the graphs, Cypher was used as a querying language, and Gephi was used for visualization (due to the need to cope with big graphs) [7].

\section{DISSECTING THE COMPONENTS OF KBE IN PARAMETRIC DESIGN}

We concentrate on parameterized Design model in particular, and parametric CAD modeling controlled by spread sheets or KBE solutions, in our research. A parameterized CAD design may be handled in 3 ways (CAD structures are considered as parametric). For starters, you may link worksheets and CAD designs together to build parent table, which seem to be collections of core aspects generated from an unified customizable CAD form. This method may be used to work with design methods of conventional components, screws, and other connectors [8]. Secondly, KBE technologies may be linked to Digital model, allowing for the execution of rules to modify the designs. When dealing with parts and systems that are subject to change depending on client needs, this is helpful. 
A third option is to use worksheet formulae and incorporate these into the geometrical reconstruction. The spreadsheets software has become a KBE-system in this instance. We'll look at three different kinds of engineering composition: CAD modeling, worksheets, and KBE solutions, to understand how they work and how they're connected. We next set the foundation for future research in knowledge and complexity management using graph theory and filters.

Models Created Using Computer-Aided Design (CAD)

A CAD modeling may be produced in a variety of methods, and it varies depending on the CAD technology (thus the neutral CAD format is required). Despite this, Fig 2 depicts a graphical database schema that depicts the widely accepted core elements. An assembly is made up of instances of pieces that may be components or assemblies, as indicated in the figure. The term utilize in literature is different when it comes to defining what is a component and what is a part. In this case, a component represents a part that has been extracted from materials, and a part is a single composition or component with multiple components.

At least one feature is required for components, whereas assemblies may or may not contain features. Variables are bearers of basic data available such as boolean algebra, mathematics, integers, and text elements packaged with a title, and they may be found in parts and systems. Calculations (quantitative formulas with variables) [9] can be found in components and modules. Geometrical elements are the most frequent type of CAD modelling constituent. Points, arcs, lines, surfaces, and interfaces, which can be one-, two-, or three-dimensional mathematical components, make up geometrical features. Geometrical dimensions, often known as restrictions, are a type of parameter that regulates the definitions of geometrical entities.

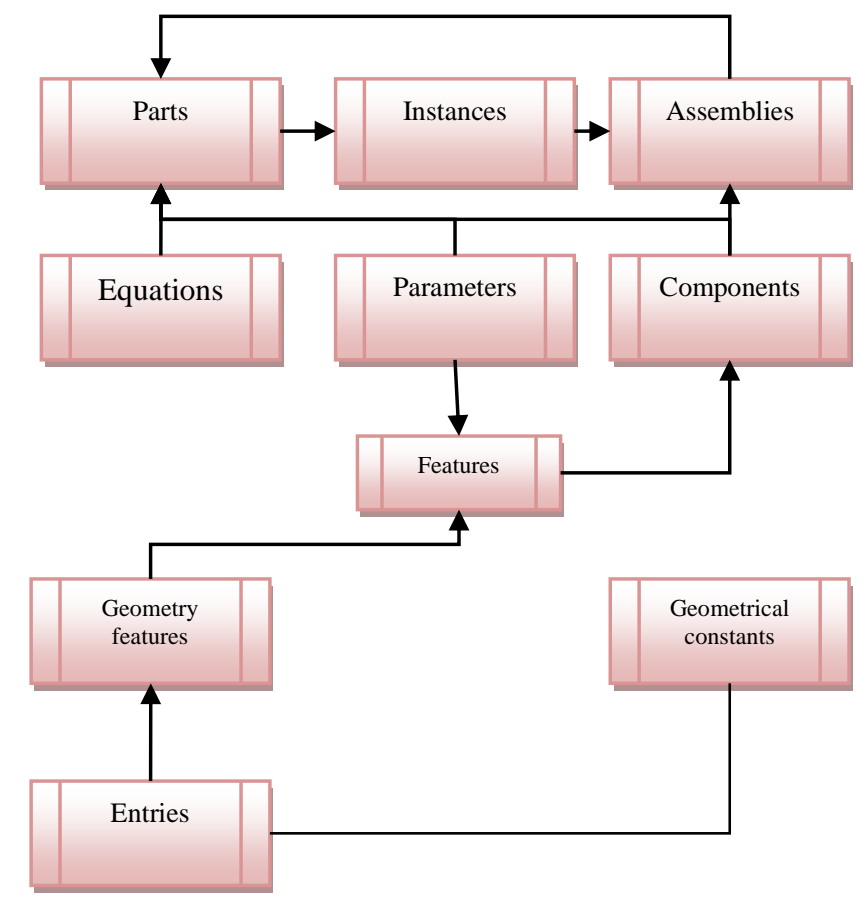

Fig 2. Data framework of the CAD model - Various connection types within the model

As shown in Fig 2, elements and components are linked via "part of" relationships. Organizations are "parts of" characteristics, whereas characteristics are "parts of" variables and expressions. A "modeling tree" is often used in CAD systems to illustrate these simple connections. Fig 3 depicts the basic modeling tree, which has a top component (Assembly 1) with three cases of components (2 Sub-Assembly 1 and 1 Sub Assembly 2). Sub Assembly 1 is comprised of two occurrences of Component 1, which is made up of Feature 1 and Feature 2. (Keep in mind the not each CAD modeling is capable of displaying the case level within the modeling tree, i.e., nodes Sub-Assembly (one), Sub Assembly 1 (two), and Sub Assembly 2 (one).

Other than "part of" relationships, CAD models include hidden relationships that engineers find fascinating while constructing the representations. The references among mathematical things communicated via geometrical dimensions or restrictions are an example of this kind of connection. Local reference are effectively managed by the geometric algorithms, while cross reference among entities belonging to distinct features result in hierarchy interdependence referred to as parent/child relationships (whereby eliminating the parent element will delete the child). The purpose of the CADmodel is another example of essential connections for engineers. 


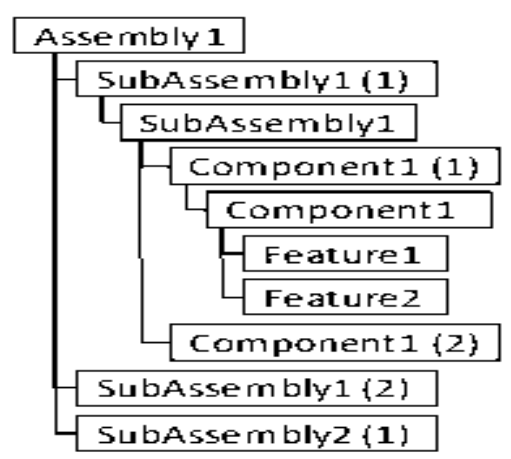

Fig 3. Framework tree within the CAD system - "Part of" relation

Most CAD systems allow for the addition of reasoning to the CAD framework based on the application of formulas that might control the parameter (e.g. the overall occurrences of parameters that repeat), mathematical dimension (e.g. sizes and topologies of the CAD framework), or mathematical connections (e.g. the Boolean flag, which can control parallelism or the aspect of perpendicularity between two different firms [10]. They are referred to as mathematical relations. If these relationships are unary expressions, they may be considered parent/child relationships. Table 2 shows how the relationships are represented in the graphs as edges.

\section{Spreadsheet}

Spreadsheets are a component of the modelling approach and are often used in structural engineering to store and handle information about the product. Spreadsheets may be used as design tables in CAD models or as part of the mathematical construction process as a "analysis" component, as previously stated. The freedom to model information is one of the reasons for using spreadsheets in the product model. The cell is the most important concept in spreadsheet apps. A graphical information framework of spreadsheets is shown in Fig 4. Worksheets include cells, and worksheets contain worksheets, as shown in Fig 4. A formula that relates to other cells may be found in a cell. Formulas work like functions, with many input cells but only one output cell that can be seen in the column to which it corresponds.

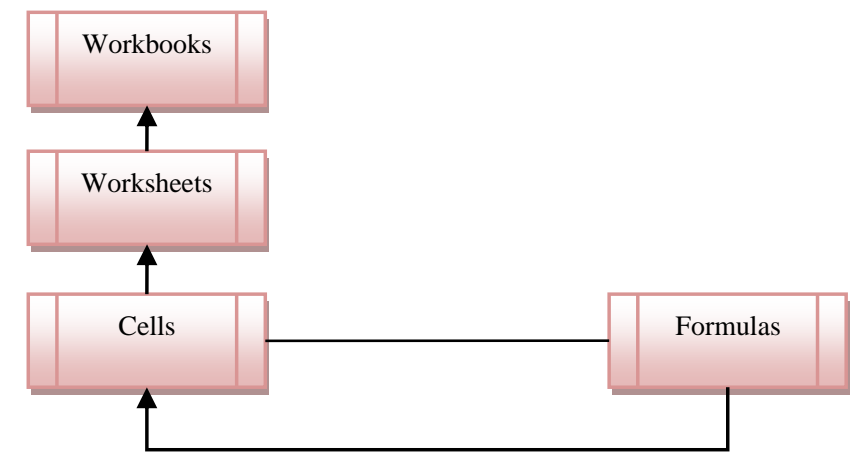

Fig 4. Data framework of the spreadsheet

We may consider possible combinations of the formulae and facts are a regulation within the KBE systems when coupled to a CAD model, per the concept of $\mathrm{KBE}$ in [11], where the iterative procedure is implemented by the data management platform (which spontaneously upgrade cells and orientations on several results whenever the connected cell transforms).

\section{Knowledge Base}

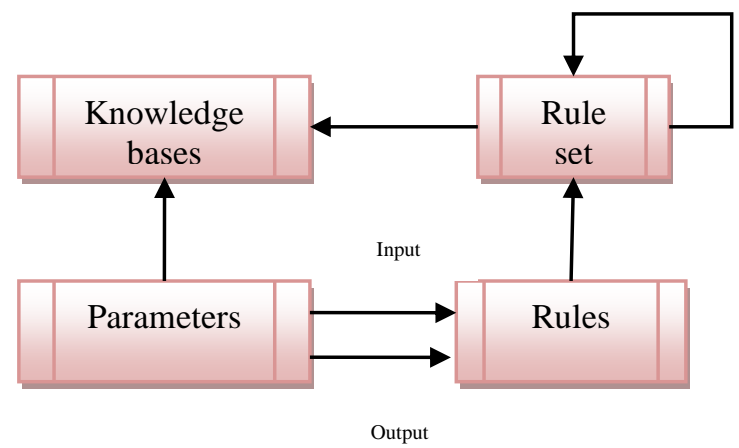

Fig 5. Data framework of knowledge base 
During the past decade, KBE networks have received popularity and have been incorporated into a number of $\mathrm{CAD}$ systems. There are also standalone KBE systems. The Rule subclass is by far the most exciting topic in a KBE-system. Rules are stored in rule sets, as shown in Fig 5, and rule sets are stored in Knowledge database. Rules are made up of variables and operate as function of one or more input representation of data variables. Because the variable class in a base of knowledge is broader than in a CAD model, the variables in Fig 2 are a subtype of the variables in Fig 5.

\section{Graphs and Filters}

CAD modelling, spread sheet, and information bases, as well as their relationships, constitute networking that can be depicted as graphs. To obtain insights into the concept, these charts can be sorted and displayed. They can also be utilized as a starting point for understanding logic, as explained in [12]. To do all of this, characteristics can be added to vertices and edges to make the chart more relevant. Table 1 lists the features associated with the vertices and edges in this article. The Edge Type property differentiates the charts provided in the study from standard model-trees in CAD systems since it allows for modelling of how objects are related. When looking at the sequence diagram in Fig 2, Fig 4, and Fig 5, you'll see five kinds of pairings, which are mentioned in Table 2.

The variables in the table's first columns are acceptable choices for the Edge Type feature, as well as descriptions for the edge.

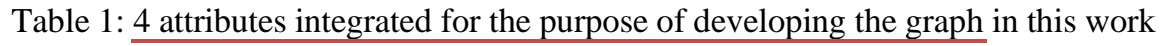

\begin{tabular}{ccc}
\hline Names & Application & Definition \\
\hline $\begin{array}{c}\text { URI (Unique } \\
\text { Resource } \\
\text { Identifiers) }\end{array}$ & Node & $\begin{array}{c}\text { File paths and } \\
\text { the inner paths } \\
\text { to the } \\
\text { represented } \\
\text { firm }\end{array}$ \\
Labels & Node and edge & $\begin{array}{c}\text { Text in the } \\
\text { chart }\end{array}$ \\
Kind of firm & Node & Firm type \\
Type of edge & Edge & $\begin{array}{c}\text { Relations to } \\
\text { Table } 2\end{array}$ \\
\hline
\end{tabular}

Table 2: 5 forms of relationships identified in the CAD system (the one indicated by * denote the type that is not directed Interlinked elements are illustrated in Fig 2 and Fig 5.

\begin{tabular}{|c|c|c|}
\hline $\begin{array}{l}\text { Type } \\
\text { relations }\end{array}$ & $\begin{array}{l}\text { Interlinked } \\
\text { constituents }\end{array}$ & $\begin{array}{l}\text { Considered } \\
\text { based on: }\end{array}$ \\
\hline Part of & Firm $\leftrightarrow$ Feature & Feature firm \\
\hline Part of & $\begin{array}{l}\text { Feature } \leftrightarrow \\
\text { Element }\end{array}$ & Element feature \\
\hline Part of & $\begin{array}{l}\text { Element } \leftrightarrow \\
\text { Assembly }\end{array}$ & $\begin{array}{l}\text { Assembly } \\
\text { instance }\end{array}$ \\
\hline Type of & $\begin{array}{l}\text { Instance } \leftrightarrow \\
\text { Part }\end{array}$ & Instance \\
\hline Connections & $\begin{array}{l}\text { Firm } \leftrightarrow \\
\text { Features }\end{array}$ & $\begin{array}{l}\text { Feature } \\
\text { references }\end{array}$ \\
\hline Arithmetic & $\begin{array}{l}\text { Cells } \leftrightarrow \text { Cells } \\
\text { Parameters } \leftrightarrow \\
\text { Parameters }\end{array}$ & $\begin{array}{l}\text { Expression } \\
\text { formula } \\
\text { equations }\end{array}$ \\
\hline Connections* & $\begin{array}{l}\text { Firm } \leftrightarrow \text { Firm; } \\
\text { Part } \leftrightarrow \text { Part }\end{array}$ & $\begin{array}{l}\text { Arithmetic } \\
\text { metrics }\end{array}$ \\
\hline Spatial & Firm $\leftrightarrow$ Firm & Locations \\
\hline
\end{tabular}

The intertextuality of data is realized by the filtration of data in the graph, and because of the creative aspect of architecture, there will never be a set of global filtering; nevertheless, some filtration may be more generic or more commonly used than others. Here are three pairs of filtering that we recommend. The first pair of filtering consists of extracting nodes of grade 1 that are of the kinds Geometrical Dimensions, Parameters, and Cells (i.e., nodes indicating things that influence the architecture). The objects indicated by the recovered nodes of such filtration are the CAD-entry model's point; altering any of their variables has an effect on the design.

These are referred to as design specifications in daily terminology. Filtration on the "part of" and the "kinds of" connections provides a model architecture as illustrated within the CAD model (it defines the integration of the assembly tree and the parts trees that could be applied in the evaluation of the intricacies of the computer-assisted designs [13]. The interface between the Computer aided and layout databases are produced by the third period of filtration, which entails screening edge on Exterior links. The third recommended group of filtering is to add lines of category "Mathematical," which gives the whole set of reasoning for the Computer aided. The product model's logic part is represented by the node generated by that filtering. 


\section{Rendering Graphs}

They can be studied using the concept in the preceding chapters to display charts to get a continuous view of the architectural contents of Computer aided and their related spreadsheet and information databases. The assessment is performed using three methods in our deployment. The first one navigates the Computer aided model and the framework to effectively access the nodes. Another technique evaluates the various worksheets interconnected to the Digital model in order to identify nodes both within the spreadsheets and in relation to the design. To identify nodes and edges, the third cycles through all criteria and related variables. The outcome of these techniques is demonstrated in a short illustration.

Let's pretend we have a Computer model of a container with holes in it (see left of Fig 6). There are only two extrusion aspects in the following pattern: one for the container and one for the holes. The two characteristics are built on parameter drawings, which are displayed in the modeling structure (see the center of Fig 6). A layout database determines the container's measurements, such as altitude, height, and depth, which are determined by columns in a linked spreadsheets. There are two setups (or commodity examples) that have been specified (Default and Config1). The first configuration's system of principles is governed by a "reference level," which acts as a kind of "scaling" for all three factors and is placed in the column whereas a different template is on the right side as stipulated in Fig 6 below.

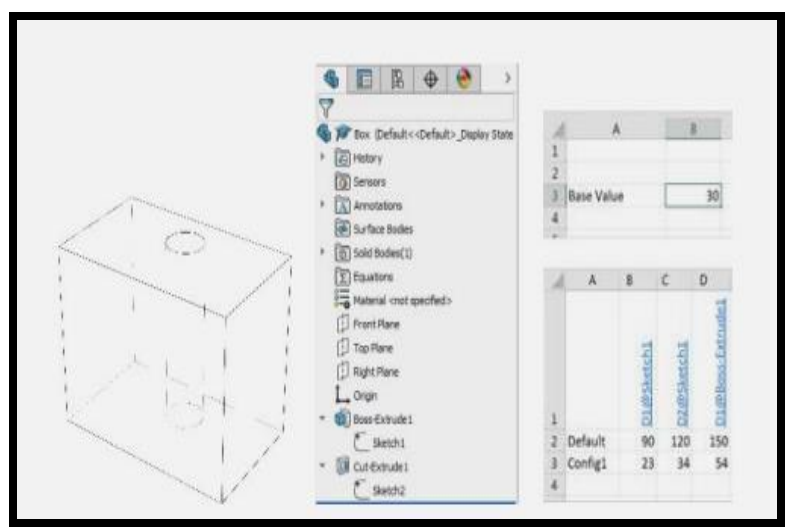

Fig 6. CAD framework in a box with hole against the framework tree and connected worksheet,

When the methods are applied to the simplest box-with-hole instance, a structure with 22 vertices and 28 links is produced. The Yifan Hu layout method is used to illustrate the structure in Figure 8. Due to the use of abbreviated identifiers on the vertices, there appear to be duplicated vertices (for example, two D1) (Label attribute). Because the URI makes each node distinct, publishing the entire identifiers would eliminate the seeming duplication.

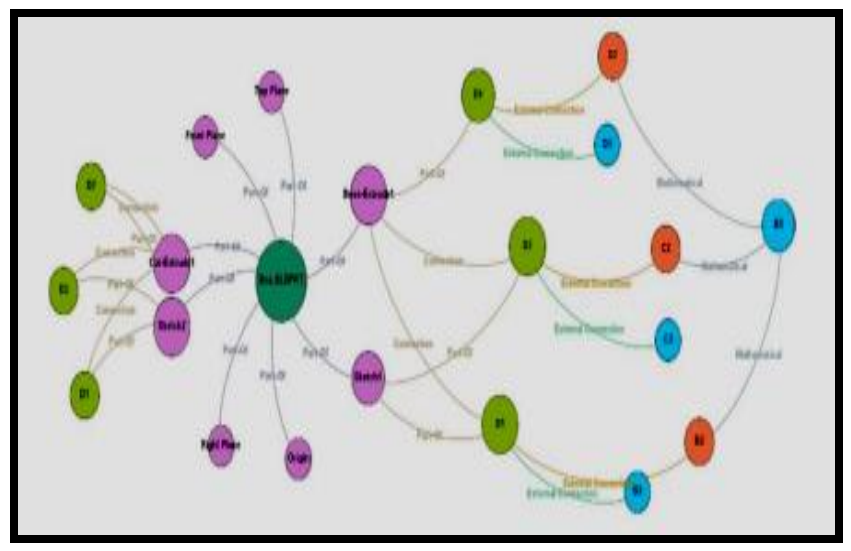

Fig 7. Graph representaiton showing the connection within the CAD framework

Fig 7 shows the vertices D1, D2, and D3 (represented by green color) on the left B3, B2 and Bi (represented by blue color) on the right after implementing a filtration to recover architectural variables. This is significant since it implies that each variable in a model database represents an input variable. When a criteria is used to extract part-of links, two indicators are produced, one with the CAD model and the other with the spreadsheets. The mathematical formalism is extracted using a filtration, resulting in a network containing different nodes (B3 (bluish), D2 (orange), C2 and B2, which signify the reasoning element of the organizational descriptions.

The following two chapters detail 3 case studies in which business information was obtained utilizing the tools and techniques discussed thus far. The first case study shows how graphical representation can aid in determining where a 
parameter model should be simplified to avoid failures and/or unreasonable computation times. The second situation demonstrates the situation's intricacy, since even a seemingly small part can communicate a large amount of design documentation, resulting in a very complicated structure that would demand sophisticated yet basic filter abilities.

The case study scenario shows how the intricacy assessment of the parent/child diagram in the Digital model may be used to extract information about CAD model integrity. This form of graphs represents a portion of the 5 kinds of Computer aided connections found by our research, and it was used to teach professionals how to create Computer aided that can be utilized and maintained effectively using spreadsheet and KBE systems [15].

\section{CONCLUSIONS}

This paper serves as a starting point for expressing engineering experience in market segments using connectivity viewpoint on technology. It was demonstrated that diagrams may be displayed and processed to fit the connectivity understanding idea by evaluating the elements in domain knowledge in KBE system, CAD modeling, and spreadsheets. We discussed connectivity, filtration, ambient, analytics, and conduits. Even if constructivist theory is a theoretical concept of knowledge, this study shows that it is feasible to acquire such a mentality in order to improve the way technical information is handled and to keep it current. The suggested techniques are far from complete, and considerable work has to be done before they can be made widely accessible to engineers in multinational corporations. Nevertheless, the classifiers and methodologies discussed in this work can already be used to improve product prototype knowledge by identifying points of entry (design parameters), allowing engineers to investigate production plan and retrieve a merchandise modified version master, gaining a comprehensive knowledge and the capability to construct the logical model of data integrated in the based on knowledge, CAD framework, worksheets, and instructing knowledge engineering regarding the manner in which CAD frameworks can be established so that the logical framework could be retraced. This aids manufacturers in managing and repurposing engineering knowledge as a valued asset.

\section{References}

[1]. L. Lai, "A knowledge engineering approach to knowledge management", Information Sciences, vol. 177, no. 19, pp. 4072-4094, 2007. Doi: 10.1016/j.ins.2007.02.028.

[2]. R. Marcus, "Knowledge engineering: Expert systems and information retrieval", Information Processing \& Management, vol. 25, no. 4, pp. 454-456, 1989. Doi: 10.1016/0306-4573(89)90073-3.

[3]. P. Quezada-Sarmiento and L. Enciso, "Knowledge Representation and Ontological Model based on Software Engineering Body of Knowledge as a tool to Evaluate Professional and Occupational Profiles", Journal of Information Systems Engineering \& Management, vol. 1, no. 3, 2016. Doi: $10.20897 /$ lectito.201636

[4]. T. Thamaraimanalan, M. Mohankumar, S. Dhanasekaran, "Experimental analysis of intelligent vehicle monitoring system using Internet of Things (IoT)," EAI Endorsed Transactions on Energy Web, p. 169336, Jul. 2018.

[5]. S. Pollitt, "Knowledge engineering: Expert systems and information retrieval", International Journal of Information Management, vol. 9, no. 3, pp. 215-217, 1989. Doi: 10.1016/0268-4012(89)90012-1.

[6]. E. Métais, "Enhancing information systems management with natural language processing techniques", Data \& Knowledge Engineering, vol. 41, no. 2-3, pp. 247-272, 2002. Doi: 10.1016/s0169-023x(02)00043-5.

[7]. R. Dillnut, "Surviving the information explosion [knowledge management]", Engineering Management, vol. 16, no. 1, pp. 39-41, 2006. Doi: 10.1049/em:20060109.

[8]. B. van den Hooff and M. Huysman, "Managing knowledge sharing: Emergent and engineering approaches", Information \& Management, vol 46, no. 1, pp. 1-8, 2009. Doi: 10.1016/j.im.2008.09.002.

[9]. D. Detmer, "Engineering information technology for actionable information and better health", Information Knowledge Systems Management, vol. 8, no. $1-4$, pp. 107-118, 2009. Doi: 10.3233/iks-2009-0138.

[10]. J. de Vasconcelos and Á. Rocha, "A special section on knowledge management and engineering", International Journal of Information Management, vol. 37, no. 1, p. 1460, 2017. Doi: 10.1016/j.ijinfomgt.2016.05.011.

[11]. T. Arumugam, S. Mohan, C. R. Anandakumar"Knowledge Management: Factors Influencing Successful Innovation", Information and Knowledge Management, 2019. Doi: 10.7176/ikm/9-1-03.

[12]. S. Parsons and A. saffiotti, "Information processing and the management of uncertainty", The Knowledge Engineering Review, vol. 10, no. 1, pp. 83-88, 1995. Doi: 10.1017/s0269888900007311.

[13]. J. Elam and J. Henderson, "Knowledge engineering concepts for decision support system design and implementation", Information \& Management, vol. 6, no. 2, pp. 109-114, 1983. Doi: 10.1016/0378-7206(83)90004-6.

[14]. A. H and A. R, Eds., "Cognitive Social Mining Applications in Data Analytics and Forensics," Advances in Social Networking and Online Communities, 2019

[15]. W. Rouse and W. Compton, "Systems engineering and management", Information Knowledge Systems Management, vol. 8, no. 1-4, pp. 231240, 2009. Doi: 10.3233/iks-2009-0139. 